\title{
Drug Utilization Evaluation of Vancomycin Among Hospitalized Patients in Internal Medicine Wards of Tikur Anbessa Specialized Hospital
}

\author{
Bereket Zeleke $^{1}$, Ephrem Engidawork ${ }^{2}$ \\ ${ }^{1}$ College of Medicine and Health Sciences, Madawalabu University, Bale-Goba, Ethiopia \\ ${ }^{2}$ Department of pharmacology and clinical pharmacy, Addis Ababa University, Addis Ababa, Ethiopia
}

Email address:

beki0979@gmail.com (B. Zeleke),ephrem.engidawork@gmail.com (E. Engidawork)

To cite this article:

Bereket Zeleke, Ephrem Engidawork. Drug Utilization Evaluation of Vancomycin Among Hospitalized Patients in Internal Medicine Wards of Tikur Anbessa Specialized Hospital. American Journal of Health Research. Vol. 3, No. 6, 2015, pp. 333-337.

doi: 10.11648/j.ajhr.20150306.13

\begin{abstract}
Background: Vancomycin use is considered inappropriate in most hospitals. A particular concern is the recent emergence vancomycin-resistant enterococci (VRE) which is related to the potential for nosocomial transmission, the lack of antibiotics to treat infections caused by this organism, and the possibility that the vancomycin-resistant genes present in VRE can be transferred to other gram-positive microorganisms such as S. aureus. The objective of this study was to evaluate the utilization of vancomycin among hospitalized patients in internal medicine wards of Tikur Anbessa Specialized Hospital (TASH). Methods: A retrospective cross-sectional chart review was carried out to evaluate the utilization of vancomycin among hospitalized patients with vancomycin therapy between April 2012 to 2013. Results: One hundred twenty five patients were enrolled in the study, consisting of 60 males and 65 females. The age range was 13 to 80 years. Of all courses, $88.8 \%$ were considered to be initially appropriate, but this decreased to $6.4 \%$ after vancomycin initiation. Continued empiric use of vancomycin without further evidence of gram-positive infections accounted for the majority of inappropriate use. The major dosing regimen employed was $1 \mathrm{~g}$ every $12 \mathrm{~h}$ in $99(79.2 \%)$ of treatments. Vancomycin dose was not adjusted or adjusted inadequately for $62(96.5 \%)$ of the patients with increased serum creatinine. Furosemide was the most frequently identified potentially interactive drug. Conclusion: Although the initial indication for vancomycin use was generally appropriate, failure to continue this antibiotic as per HICPAC criteria and inappropriate dosing adjustment based on MDRD CrCl level were common challenges identified.
\end{abstract}

Keywords: Vancomycin, Drug Use Evaluation, Hicpac Guideline, Dosage, Interaction

\section{Introduction}

Vancomycin is a member of a class of antibiotics referred to as glycopeptides. These antibiotics interfere with peptidoglycan biosynthesis by binding to the terminal Dalanyl-D-alanine residue of the peptidoglycan precursor ${ }^{[1]}$.

Vancomycin use is considered inappropriate in most hospitals. A particular concern is the recent emergence of $\operatorname{VRE}^{[2]}$.The number of infections caused by VRE that have been reported to the Center for Disease Control and Prevention's (CDC's) National Nosocomial Infections Surveillance system was $40 \%$ in 2012 among US Hospitals. VRE are estimated to be responsible for 10 to $30 \%$ of all nosocomial infections in US ${ }^{[3]}$. The mounting emergence of VRE has resulted in the development of guidelines for use by
Hospital Infection Control Practices Advisory Committee (HICPAC) of CDC ${ }^{[4,5]}$.

Indications for vancomycin use were classified as appropriate or inappropriate based on modified HICPAC criteria ${ }^{[6]}$. According to the guidelines, inappropriate use of vancomycin is subdivided into five; empiric therapy without risk factors, continued empiric use without further culture evidence of gram-positive infections, treatment of Methicillin susceptible Staphylococcus aureus (MSSA) infections without allergy history to beta-lactam antimicrobials, treatment in response to a single blood culture positive while other blood cultures taken during the same time-frame are negative, and use as systemic or local prophylaxis for infections ${ }^{[7]}$.

According to a national survey conducted to map antimicrobial resistance in Ethiopia, VRE accounted for around 
$8.7 \%$ of all identified enterococci species ${ }^{[8]}$. Concern about VRE is related to the potential for nosocomial transmission, the lack of antibiotics to treat infections caused by this organism, and the possibility that the vancomycin-resistant genes present in VRE can be transferred to other gram-positive microorganisms such as Staphylococcus aureus ${ }^{[3]}$.

Drug Utilization Evaluation (DUE) is an effective mechanism to identify individual variability in drug use and to promote interventions that will improve positive patient outcomes ${ }^{[9,10,11]}$.

Vancomycin is an antibiotic of growing importance in the treatment of hospital acquired infections, with particular emphasis on its value in the fight against MRSA. However, its usage profile must be evaluated to assure maximum benefit and minimum risk. Such studies are lacking in Ethiopia thus it would be prudent to assess whether vancomycin is used appropriately in a cost effective manner so as to improve patient outcomes.

\section{Materials and Methods}

The study was conducted in internal medicine wards of TASH which is located in Addis Ababa, Ethiopia. TASH was established in 1962 with the aim of improving the provision of health services in Ethiopia. A retrospective cross-sectional chart review was carried out to evaluate the utilization of vancomycin among hospitalized patients with vancomycin therapy in in internal medicine wards of TASH between April 2012 to 2013.

Medical records (charts) had been reviewed using individual patient data abstraction format. Both the initial indication as empiric therapy and vancomycin prescription following the availability of culture and sensitivity results was assessed. Information that was collected included demographic data, renal function parameters, admission diagnosis, type of infection, evidences for empiric vancomycin therapy, the judgment on whether vancomycin prescription was empirical or based on culture, history of allergies, concurrent antimicrobials and nephrotoxic drugs, vancomycin dosage, route of administration, duration of vancomycin therapy, culture and sensitivity test results. Key informant interview was conducted with physicians from infectious disease unit to gather information like the reason behind inappropriate vancomycin use and dosing; criteria used to start and continue empiric therapy, and commonly used guidelines for prudent vancomycin use.

Analysis was done using SPSS version 20.0. The descriptive statistic methods were used to summarize data. Determination of appropriateness of dose adjustment creatinine clearance was calculated using the modification of diet in renal disease (MDRD) formula. Information from Drug.com data base was used to identify potential interaction and Chi-square $\left(\mathrm{X}^{2}\right)$ had been used to identify possible association between ages, ward units, types of indications, and outcomes after therapy and time after initial therapy with appropriateness of vancomycin use. $P<0.05$ was considered statistically significant.

\section{Results}

Between April 2012 and 2013, a total number of 125 patients (60 males and 65 females) received a total of 125 courses of vancomycin. There were 104 adults (mean \pm S.D. age, $37 \pm 14$ years) and 18 patients above 65 years of age. The average length of hospital stay was 20.7 days; the average length of hospital stay prior to vancomycin use was 9 days. Of the patients studied, 54.4\% died after $72 \mathrm{~h}$ of vancomycin therapy. No patient was allergic to $\beta$-lactam antimicrobials (Table 1).

Table 1. Socio-demographic and disease characteristics of the study participants.

\begin{tabular}{lll}
\hline Variables & Number $(\mathbf{n = 1 2 5})$ & Percentage $(\%)$ \\
\hline Age of patients(yrs.) & & \\
$\leq 13$ & 3 & $2.4 \%$ \\
$13-65$ & 104 & $83.2 \%$ \\
$\geq 65$ & 18 & $14.4 \%$ \\
Sex of patients & & \\
Male & 60 & $48 \%$ \\
Female & 65 & $52 \%$ \\
Hospital stay & & \\
$<$ two weeks & 46 & $36.8 \%$ \\
$\geq$ two weeks & 79 & $63.2 \%$ \\
Outcome & & \\
Death & 68 & $54.4 \%$ \\
Discharge & 57 & $45.6 \%$ \\
\hline
\end{tabular}

The most frequent indications to the vancomycin use were pneumonia (54.40\%) and primary sepsis (23.20\%) (Fig1).

After starting vancomycin as an empiric therapy, the drug use was consistent with HICPAC guidelines only in $8(6.4 \%)$ patients. Of these, majority $6(75 \%)$ had discontinued empirical use for presumed infections in patients yielding subsequent culture negative results. Continued empiric use without further evidence of gram-positive infections accounted for $94.8 \%$ of inappropriate vancomycin use, according to CDC recommendations (Table 2).

Renal function was monitored to almost all of the patients, however, the doses were not adjusted correctly or not at all adjusted according to MDRD $\mathrm{CrCl}$ formula for $62(96.9 \%)$ of patients that had renal failure (Table 3).

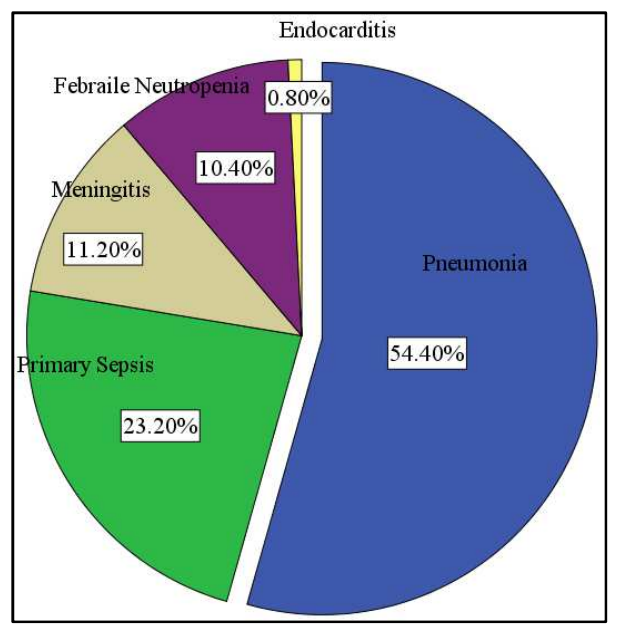

Figure 1. Indications for vancomycin use among the study participants. 
Table 2. Vancomycin use based on HICPAC guideline among the study participants.

\begin{tabular}{|c|c|}
\hline Initial treatment type & $\mathbf{N}(\%)$ \\
\hline Empirical & $125(100 \%)$ \\
\hline Microbiologically documented & $0(0 \%)$ \\
\hline Vancomycin use & $\mathrm{N}(\%)$ \\
\hline Consistent with HICPAC guideline & $8(6.4 \%)$ \\
\hline $\begin{array}{l}\text { Discontinued empirical use for presumed infections in } \\
\text { patients yielding subsequent culture negative results }\end{array}$ & $6(75 \%)$ \\
\hline $\begin{array}{l}\text { Continued empiric use for presumed infections in patients } \\
\text { yielding subsequent cultures positive for } \beta \text {-lactam-resistant } \\
\text { gram-positive organisms }\end{array}$ & $1(12.5 \%)$ \\
\hline $\begin{array}{l}\text { Discontinued empirical use for in patients yielding } \\
\text { subsequent cultures positive for } \beta \text {-lactam-susceptible gram- } \\
\text { positive organisms without } \beta \text {-lactam allergy }\end{array}$ & $1(12.5 \%)$ \\
\hline Inconsistent with HICPAC guideline & $117(93.6 \%)$ \\
\hline Empiric therapy without risk factors & $3(2.6 \%)$ \\
\hline $\begin{array}{l}\text { Continued empiric use without further evidence of Gram- } \\
\text { positive infections }\end{array}$ & $111(94.8 \%)$ \\
\hline
\end{tabular}

Table 3. Appropriateness of vancomycin dosing adjustment according to $M D R D C r C l$ equation among study participants with renal disease.

\begin{tabular}{lll}
\hline Adjustment & N (\%) \\
\hline Not required & & $61(48.8 \%)$ \\
Required & & $64(51.2 \%)$ \\
& Correctly adjusted & $2(3.1 \%)$ \\
& Incorrectly adjusted & $22(34.4 \%)$ \\
& No adjustment made & $40(62.5 \%)$ \\
\hline
\end{tabular}

When dose used was disaggregated by $\mathrm{CrCl}, 1 \mathrm{~g}$ every 12 $\mathrm{h}$ was commonly employed in patients with normal or nearnormal renal function $(\mathrm{CrCl}>65 \mathrm{ml} / \mathrm{min})$ and this was found to be appropriately used in almost all patients in this category. Inappropriate dose used among these patients was small and largely attributed to under dosing. In patients with low renal function, although an attempt was made to adjust the dose, the adjustment was incorrect in majority of the cases. Doses used for the treatment of greater proportion of patients with moderately decreased $\mathrm{CrCl}(30-59 \mathrm{ml} / \mathrm{min})$ were not adjusted (Fig 2).

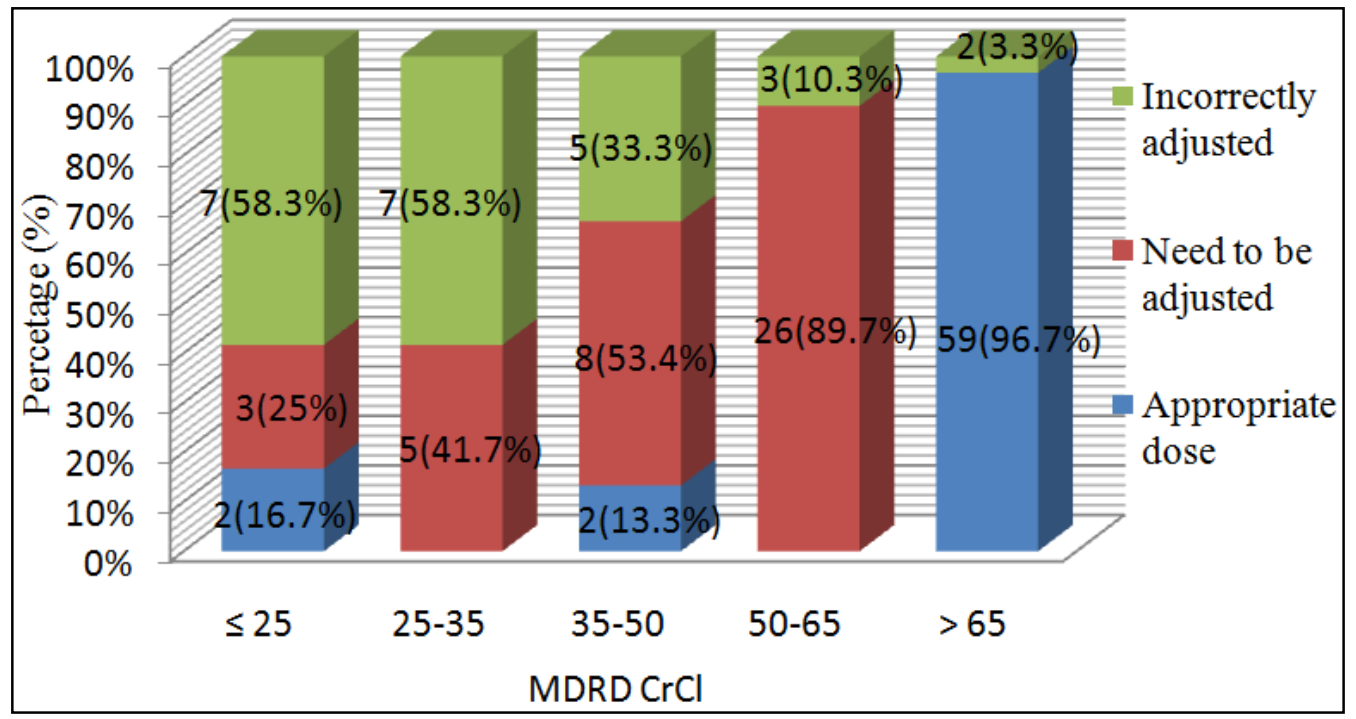

Figure 2. Vancomycin dosing pattern based on MDRD CrCl calculations among study participants with renal disease.

Table 4. Bivariate analyses of risk factors for inconsistent vancomycin use, according to the CDC criteria (HICPAC), among study participants.

\begin{tabular}{|c|c|c|c|c|}
\hline \multirow[t]{2}{*}{ Variables } & & \multicolumn{2}{|c|}{$\begin{array}{l}\text { Use of vancomycin as per } \\
\text { HICPAC guidelines }\end{array}$} & \multirow[t]{2}{*}{ P-value } \\
\hline & & Inappropriate & Appropriate & \\
\hline \multirow{2}{*}{$\begin{array}{l}\text { Age of } \\
\text { patients }\end{array}$} & $<65$ & 100 & 8 & \multirow{2}{*}{0.000} \\
\hline & $\geq 65$ & 17 & 0 & \\
\hline \multirow{2}{*}{$\begin{array}{l}\text { Internal } \\
\text { medicine } \\
\text { units }\end{array}$} & ICU & 26 & 1 & \multirow[b]{2}{*}{0.20045} \\
\hline & Non-ICU & 91 & 7 & \\
\hline \multirow{2}{*}{$\begin{array}{l}\text { Type of } \\
\text { indication }\end{array}$} & Pneumonia & 63 & 5 & \multirow{2}{*}{0.10027} \\
\hline & Others & 54 & 3 & \\
\hline \multirow{2}{*}{$\begin{array}{l}\text { Time after } \\
\text { therapy } \\
\text { initiation }\end{array}$} & Before $24 \mathrm{hrs}$ & 14 & 111 & \multirow[b]{2}{*}{0.000} \\
\hline & After $72 \mathrm{hrs}$ & 117 & 8 & \\
\hline
\end{tabular}

Out of the total charts reviewed in the present study, 52 contained at least one potential drug-drug interaction (D-DI) and four potential D-DIs were counted 57 times without any special vancomycin dose adjustment. Furosemide (Lasix) and
Tenofovir (TDF) were the most frequently and the only major interactive drugs identified. Renal diseases account for 45 out of 58 potential drug-diseases interactions

After several factors were analyzed, it was found out only age and time after initiation of the drug to have association with inappropriate use of vancomycin (Table 4).

\section{Discussion}

Vancomycin is used in the treatment of serious infections caused by susceptible organisms resistant to $\beta$-lactam antibiotics like MRSA or in individuals with serious allergy to penicillin ${ }^{[9]}$. The most frequent indications to vancomycin use in our study were pneumonia (54\%) followed by primary sepsis. In previous studies, Febrile neutropenia and primary sepsis were the two common indications to which vancomycin were most frequently used and they accounted for $87.9 \%$ and $74.5 \%$, respectively, of the total number of 
reasons for vancomycin used ${ }^{[12,13]}$. According to the result obtained through interview with physicians from infectious disease unit, the high prevalence of vancomycin use for pneumonia is suggestive of pneumonia to be the most common hospital acquired infection in the internal medicine wards of the hospital.

Although rational use is mandatory, high prevalence of MRSA may raise the consumption of glycopeptide for empiric therapy in hospitals. All of the patients in this study received empiric treatment for suspected gram-positive infections. This is higher than rates reported by other researchers which range from $71 \%$ and $98 \%{ }^{[7,12,13]}$. This outcome is suggestive of high prevalence of MRSA in TASH ${ }^{[8]}$, probably necessitated a high empiric vancomycin use and independence of vancomycin initiation and continuation with culture results because of accessibility and quality issue.

Inappropriate use of vancomycin is a major contributing factor in the emergence of VRE ${ }^{[14,15]}$. Generally vancomycin use was considered inconsistent with HICPAC guidelines in $117(93.6 \%)$ patients. Of the total inappropriate vancomycin use, $94.8 \%$ were due to continued empiric use without further evidence of gram-positive infections by culture results. This is higher than rates reported by other researchers which varied between $46 \%$ and $95.4 \%{ }^{[7,12,13]}$. According to the key informant interview, continuation of vancomycin empiric therapy in the hospital doesn't depend on the microbiological data due to availability and quality issue. The key informant interview also highlighted that few of the physicians in the hospital were aware of the HICPAC recommendations.

Glomerular filtration rate (GFR) is the best index of kidney function. Mathematical estimations of GFR, based on serum creatinine, are clinically useful methods to follow renal function ${ }^{[16]}$. Absence of patient weight in the medical chart limited us to calculate $\mathrm{CrCl}$ based on Cockcroft Gault (CG) equation which is used by most researchers for vancomycin dosing evaluation. As use of CG and MDRD provided discordant estimations ${ }^{[16]}$, the results of dose adjustment based on MDRD $\mathrm{CrCl}$ in our study was compared with the previous studies which were evaluated based on similar equation. According to $\mathrm{MDRD} \mathrm{CrCl}$ calculation, vancomycin dose was not corrected or corrected inadequately for $96.5 \%$ of patients that had renal failure. This is also higher than rates reported by other researchers which range from $11.5 \%$ to $60 \%{ }^{[7,12,13]}$. These findings suggest that vancomycin dosing among patients with renal impairment in our hospital was not calculated based on $\mathrm{CrCl}$ results even though the physicians' interview revealed GFR is used to adjust vancomycin dose in internal medicine wards of TASH.

Bivariate analysis showed that the risk factors associated with inappropriate vancomycin use, according to the CDC criteria, were patients above 65 years old, time after $72 \mathrm{~h}$ of empiric vancomycin therapy. In our analysis, patients those who were hospitalized in units other than ICUs did not have risk factors associated with inappropriate use of vancomycin as compared to ICU patients. The high incidence of inappropriate vancomycin use after $72 \mathrm{~h}$ of therapy in our study is associated with continued empiric use without further evidence of gram-positive infections. According to a previous study, patients aged less than 60 , non-ICU patients and those who did not present neutropenia were the principal groups at risk of inappropriate use as per the CDC criteria ${ }^{[2]}$.

\section{Conclusion}

Although the initial indication for vancomycin use was generally appropriate, failure to continue this antibiotic as per HICPAC criteria and inappropriate dosing adjustment based on MDRD $\mathrm{CrCl}$ level were common challenges identified in internal medicine wards of TASH.

\section{References}

[1] Maciej C, Asok K. Vancomycin-resistant Enterococcus. Ann Acad Med Singapore 2008; 37: 861-9.

[2] Moacyr S, Luci C, Alexandre R, et al. Analysis of vancomycin use and associated risk factors in a university teaching hospital a prospective cohort study. BMC Infectious Diseases 2007; 7(88): 1-6.

[3] Center of Disease Control and Prevention guideline for Antibiotic Resistance Threats in the United States 2013.

[4] Centers of Disease Control and Prevention for management of Multidrug-Resistant Organisms in Healthcare Settings 2007.

[5] Centers for Disease Control and Prevention/Healthcare Infection Control Practices Advisory Committee (HICPAC). Draft Guidelines for Environmental Infection Control in Healthcare Facilities in Atlanta 2001.

[6] Kevin G, Gerald S, David W, et al. Vancomycin Prescribing Practices in Hospitalized Chronic Hemodialysis Patients. American Journal of Kidney Diseases 2000; 35: 64-68.

[7] Daniela O, Marli S, Renato S. Vancomycin Use in a Hospital with High Prevalence of Methicillin-Resistant Staphylococcus aureus: Comparison with Hospital Infection Control Practices Advisory Committee Guidelines. BJID 2007; 11(1): 53-56.

[8] Drug Administration and Control Authority of Ethiopia in collaboration with Management Sciences for Health, Strengthening Pharmaceutical Systems (MSH/SPS) August 2009.

[9] Wasam L, Mehreen F, Fatima A, Khawaja T. Drug Utilization Evaluation of Vancomycin in Teaching Hospitals of Lahore. J Pharm Sci \& Res 2012; 4(2): 1728-33.

[10] Jyothi K, Jagadish Babu D. Drug Utilization Evaluation of Cephalosporins in General Medicine Units of Rural Tertiary Care Hospital. Int J Curr Pharm Res 2012; 4 (2): 88-91.

[11] Navarro, Robert. Drug Utilization Review Strategies. Managed Care Pharmacy Practice 2008; 8: 215-229.

[12] Joyce H, Donald J, Benjamin S, et al. Vancomycin utilization at a teaching hospital in Hong Kong. Am J Health-Syst Pharm 2001; 58: 2167-9.

[13] Afsaneh V, Aziz J, Sakineh S. Vancomycin Utilization Evaluation at Hematology-Oncology Ward of a Teaching Hospital in Iran. Iranian Journal of Pharmaceutical Research 2012; 11 (1): 163-170. 
[14] Surendra K, Leanne H, Gillian L, et al. Prevalence and risk factors for VRE colonization in a tertiary hospital in Melbourne, Australia. Antimicrobial Resistance and Infection Control 2012, 1: 31-7.

[15] Alison G, Kent A, Craig A, et al. Clinical Practice Guideline for the Use of Antimicrobial Agents in Neutropenic Patients with Cancer. Clinical Infectious Diseases 2011; 52(4): 56-93.

[16] Jagbir G, Rhonda M, Ognjenka D, Adeera L. Use of GFR equations to adjust drug doses in an elderly multi-ethnic group. Nephrol Dial Transplant 2007; 22: 2894-9. 